Original Paper

\title{
SIRT1 Involved in the Regulation of Alternative Splicing Affects the DNA Damage Response in Neural Stem Cells
}

\author{
Guangming Wang ${ }^{a, b, c}$ Fangce Wang ${ }^{b}$ Jie Ren ${ }^{a}$ Yue Qiu ${ }^{a}$ Wenjun Zhang \\ Shane Gao ${ }^{a}$ Danjing Yang ${ }^{a}$ Zhigang Wange Aibin Liang ${ }^{b}$ Zhengliang Gaoc,d \\ Jun $\mathrm{Xu}^{\mathrm{a}}$
}

aEast Hospital, Tongji University School of Medicine, Shanghai, ${ }^{b}$ Department of Hematology, Tongji Hospital of Tongji University School of Medicine, Shanghai, 'Shanghai Tenth People's Hospital, Tongji University School of Medicine, Shanghai, dAdvanced Institute of Translational Medicine, Tongji

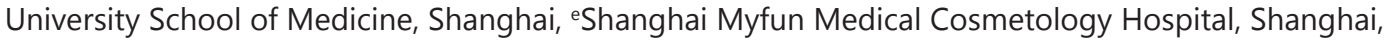
China

\section{Key Words}

Sirt1 • Alternative splicing $•$ DNA damage $•$ Neural stem cell

\begin{abstract}
Background/Aims: Alternative splicing and DNA damage exhibit cross-regulation, with not only DNA damage inducing changes in alternative splicing, but alternative splicing itself possibly modulating the DNA damage response (DDR). Sirt1, a prominent anti-aging player, plays pivotal roles in the DDR. However, few studies have examined alternative splicing with DNA damage in neural stem cells (NSCs) and, in essence, nothing is known about whether SIRT1 regulates alternative splicing. Hence, we investigated the potential involvement of Sirt1mediated alternative splicing in the NSC DDR. Methods: Genome-wide alternative splicing profiling was performed upon DNA damage induction and SIRT1 deletion. Results: DNA damage caused genome-wide changes in alternative splicing in adult NSCs and Sirt1 deficiency dramatically altered DDR-related alternative splicing. In particular, extensive alternative splicing changes in DDR-related processes such as cell cycle control and DNA damage repair were observed; these processes were dramatically influenced by Sirt1 deficiency. Phenotypically, Sirt1 deficiency altered the proliferation and DNA repair of adult NSCs, possibly by regulating alternative splicing. Conclusion: SIRT1 helps to regulate alternative splicing, which itself affects the DDR of NSCs. Our findings provide novel insight into the mechanisms underlying the DDR in stem cells.
\end{abstract}




\section{Cellular Physiology Cell Physiol Biochem 2018;47:657-669 \begin{tabular}{l|l} 
DOI: 10.1159/000491893 & and Biochemistry \\
Published online: July 19, 2018 & $\begin{array}{l}\text { 2018 The Author(s). Published by S. Karger AG, Basel } \\
\text { www.karger.com/cpb }\end{array}$
\end{tabular} \\ Wang et al.: SIRT1 in the Alternative Splicing of Neural Stem Cells}

\section{Introduction}

Neural stem cells (NSCs) respond differently to different levels of DNA damage. In a previous study of cultured NSCs, we found that low levels of DNA damage were rapidly repaired and that high levels led to apoptosis, but that moderate levels persisted for a long time [1]. Other studies also showed that DNA damage could affect NSC proliferation, differentiation, senescence, and apoptosis [2-8]. However, the mechanisms involved in the regulation of these processes are complex and remain unclear.

Alternative splicing (AS) is a universal biological event that occurs in cells during normal development and under various stresses. DNA damage can alter the gene expression, subcellular localization, and protein modification of splicing factors, which ultimately affect the production of components that control cell fate through splicing [9-14]. Previous work has indicated that AS of genes involved in DNA damage response (DDR)-related processes, such as DNA repair, cell cycle control, and apoptosis, are extensively modulated by DNA damage [15]. On the other hand, deficiencies in some splicing factors can also induce DNA damage $[16,17]$, which suggests a close cross-regulation between AS and DNA damage.

The $\mathrm{NAD}^{+}$-dependent deacetylase SIRT1 plays important anti-aging roles in tissues such as the brain, heart, liver, and muscle by modulating the activity of some key components critically involved in regulating the cell cycle, apoptosis, and DNA repair [18, 19]. In the central nervous system, SIRT1 regulates NSC self-renewal, differentiation, and apoptosis by deacetylating cell fate commitment factors such as transcription factors, Notch-Hes1, retinoic acid, and p53 [20]. Although the classic DNA repair-related targets of SIRT1, such as WRN, Ku70, NBS1, and RAD51, have been known for a long time [21-24], recent investigations also revealed a series of factors, such as DBC1, HIPK2, and TopBP1, that influence DNA repair through the modulation of SIRT1 activity [25-27].

The mechanisms of the DDR in NSCs have largely been elucidated but remain unclear; the role of SIRT1 in DNA repair is well studied but still needs further exploration. However, studies of the impact of SIRT1 on AS are nearly absent despite the considerable importance of SIRT1 and AS in the DDR. Here, with the widely used probabilistic framework called MISO (Mixture of Isoforms), we performed profiling analyses of AS events in adult NSCs under DNA damage induction and SIRT1 deletion, aiming to elucidate how the DDR is potentially regulated by SIRT1 control of AS processes. We found that widespread splicing events occurred in adult NSCs and that numerous biological processes were affected by DNA damage and SIRT1 deficiency at the AS level. Along with the observation of overrepresentation of splicing changes in genes involved in regulation of transcription, chromatin modification, RNA processing, and splicing, we discovered that DDR-related processes such as cell cycle control and DNA damage repair were affected by SIRT1 deficiency. We also verified the phenotypic changes in proliferation and repair ability introduced by SIRT1 ablation in adult NSCs.

\section{Materials and Methods}

\section{NSC-specific SIRT1 KO mouse generation}

Sirt $1^{c o / c o}$ mice (JAX008041) and NestinCreER ${ }^{T 1}$ transgenic mice (JAX003771) were purchased from the Jackson Laboratory (Bar Harbor, ME). Sexually mature Sirt $1^{c o / c o}$ and NestinCreER ${ }^{T 1}$ mice were bred to obtain

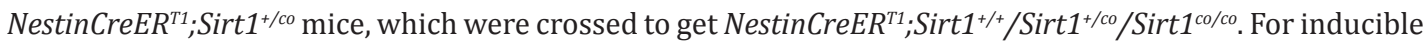
ablation of Sirt1 in adult NSCs, 2-month-old NestinCreER $R^{T 1}$; Sirt $1^{c o / c o}$ or $^{+/+}$mice were intraperitoneally injected with tamoxifen $(20 \mathrm{mg} / \mathrm{ml}$ in corn oil, $75 \mathrm{mg} / \mathrm{kg}$ body weight; Sigma-Aldrich, St. Louis, MO) for 5 successive days; 7 days later, the mice were sacrificed by cervical dislocation for tissue culture or perfused for brain slice analysis. All mice were housed in an experimental animal center at Tongji University, Shanghai, China; all animal care and experimental procedures were reviewed and approved by the Animal Committee of the school (TJmed-010-10).

\section{KARGER}




\section{Cellular Physiology Cell Physiol Biochem 2018;47:657-669 \begin{tabular}{l|l} 
DOI: 10.1159/000491893 & Ond 2018 The Author(s). Published by S. Karger AG, Basel \\
www.karger.com/cpb
\end{tabular}}

Wang et al.: SIRT1 in the Alternative Splicing of Neural Stem Cells

NSC culture

Primary mouse SVZ NSC cultures were established following a published protocol [28]. In this study, WT and SIRT1 KO NSCs were collected from three mouse brains each. After primary neurosphere formation, the cells were subcultured every 3 days. For the clone formation assay, NSCs from single-cell suspensions were seeded at a density of 500 cells per $\mathrm{ml}$ in 6-well plates; after 7 days, colonies with a diameter $>30$ $\mu \mathrm{m}$ were counted. In adherent cultures for IR treatment and immunostaining, single-cell suspensions were seeded in Matrigel (Corning Inc., Corning, NY)-coated 24-well or 6-well plates.

\section{Immunohistochemistry}

Mice were anesthetized and cardiac perfused with 4\% paraformaldehyde. Brains were dissected out, post-fixed in the same fixative for $6 \mathrm{~h}$, and successively dehydrated with 10\%, 20\%, and 30\% sucrose. The brains were frozen in Tissue-Tek optimal cutting temperature (OCT) compound (VWR, Richmond, IL) and coronally sectioned at $20 \mu \mathrm{m}$ thickness. Tissue sections were mounted on slides and then subjected to antigen retrieval in citric acid buffer $(0.01 \mathrm{M}, \mathrm{pH} 6.0)$ at $95^{\circ} \mathrm{C}$ for $15 \mathrm{~min}$. The sections were then processed with blocking buffer (3\% normal donkey serum in $0.25 \%$ Triton X-100/phosphate-buffered saline [PBS]) for $1 \mathrm{~h}$ and incubated first with primary antibodies diluted in blocking buffer for $48 \mathrm{~h}$ at $4^{\circ} \mathrm{C}$ and then with secondary antibodies for $1 \mathrm{~h}$ at room temperature. DAPI was used as a counterstain. Images were acquired and analyzed on a Leica TCS SP2 confocal microscope. Antibodies used in this procedure included nestin (mouse, Abcam, Cambridge, MA), SIRT1 (rabbit, Merck Millipore, Burlington, CA), goat anti-mouse CF488A, and goat anti-rabbit CF543 (Biotium, Fremont, CA).

\section{Immunocytochemistry}

Cell staining was performed according to the standard procedure. Briefly, cells were fixed with $4 \%$ paraformaldehyde for $10 \mathrm{~min}$, permeabilized with 0.3\% Triton X-100/PBS for $20 \mathrm{~min}$, and blocked with $3 \%$ normal donkey serum in $0.3 \%$ Triton X-100/PBS for $1 \mathrm{~h}$. Primary antibody incubation was performed overnight at $4^{\circ} \mathrm{C}$ in blocking buffer followed by 1-h secondary antibody staining at room temperature; DAPI was used for nuclear staining. Images were acquired and analyzed on a Leica TCS SP2 confocal microscope (Leica Microsystems, Wetzlar, Germany) or Nikon inverted fluorescence microscope (Nikon, Tokyo, Japan). Antibodies used in this step included $\gamma$-H2AX (rabbit; Abcam), in addition to those used for the immunohistochemistry.

\section{IR treatment}

Adherent NSCs cultured in Matrigel-coated 24-well and 6-well plates were subjected to 8 Gy radiation in an X-ray system (RX-650, Faxitron, Tucson, AZ) with the parameters set at $70 \mathrm{kV}$ for voltage and 8 min for time. The NSC cultures were then placed back in incubators until the sampling time.

\section{Western blotting analysis}

Protein samples were prepared from passaged NSC cultures derived from WT and SIRT1 KO mice. Proteins were separated by $10 \%$ stacking sodium dodecyl sulfate polyacrylamide gel electrophoresis and western blotting was performed for SIRT1 (Merck Millipore; 1:1000); actin (Sigma-Aldrich; 1:2000) was included as a loading control.

\section{RNA sequencing}

Total RNA was extracted using an RNA purification kit (Quick-RNA ${ }^{\mathrm{TM}}$ MicroPrep, R1050, Zymo Research, Irving, CA). RNA quality was analyzed by an Agilent 2100 with RNA integrity numbers above 9.0 for all samples. Next, 500-2000 ng total RNA was treated with Ribo-Zero Gold (Epicentre, Madison, WI), and mRNA was enriched using Oligo (dT) 25 Dynabeads (Life Technologies, Waltham, MA). RNA-Seq libraries were prepared and then sequenced with an Illumina HiSeq 2000. Reads with adaptors or more than $10 \%$ of $\mathrm{N}$ or containing more than $50 \%$ of bases with a quality value $\leq 5$ were filtered out using SOAPnuke. Finally, 47 to 53 million single clean reads ( $49 \mathrm{bp}$ ) were obtained for each sample.

\section{AS analysis}

FASTQ files were qualified by FastQC. Single-end sequencing reads were mapped to the mm 10 reference genome using Hisat2 (version 2.0.4) with default parameters [29]. Files in sam format were converted to 
indexed, sorted bam files with the help of the wrapper script from MISO (version 0.5.3). Aligned bam files were analyzed using the exon-centric pipeline of MISO [30]. Alternatively spliced exons ( $|\Delta \mathrm{PSI}| \geq 10 \%$, and Bayes factor $\geq 5$ ) were lifted over to the mouse genome (mm10). Sashimi plots of RNA-Seq reads were generated using the built-in sashimi plot tool in MISO. GO analyses were performed using DAVID online tools [31].

\section{Statistical analysis}

All statistical analyses except for MISO analysis were performed using the Student's t-test.

\section{Results}

AS occurs widely in adult NSCs responding to DNA damage

Because AS has a close relationship with the DDR, we compared alternative exon usage in adult subventricular zone (SVZ) NSCs with or without (CON) ionizing radiation (IR) using MISO, which assigns a "percentage spliced in" (PSI) value to each exon by estimating its abundance versus adjacent exons [30]. There were 455 differentially spliced exons between the CON and IR groups $(|\Delta \mathrm{PSI}| \geq 10 \%$ and Bayes factor $\geq 5$ ), whereas there was no significant difference in the number of events with higher inclusion for the five AS types between the $\mathrm{CON}$ and IR groups. In total, 231 events showed higher inclusion in the CON group (PSI of CON > IR) and 224 were higher in the IR group (PSI of IR > CON) (Fig. 1a), which indicated markedly different alternative exon usage between CON and IR NSCs. The 455 differentially spliced exons, which corresponded to 347 known genes, were then analyzed with Gene Ontology (GO) for Cellular Component (CC), Molecular Function (MF), and Biological Process (BP) analysis. CC and MF analyses revealed that most spliced genes were localized to the cytoplasm, nucleus, and nucleoplasm (Fig. 1b), reflecting

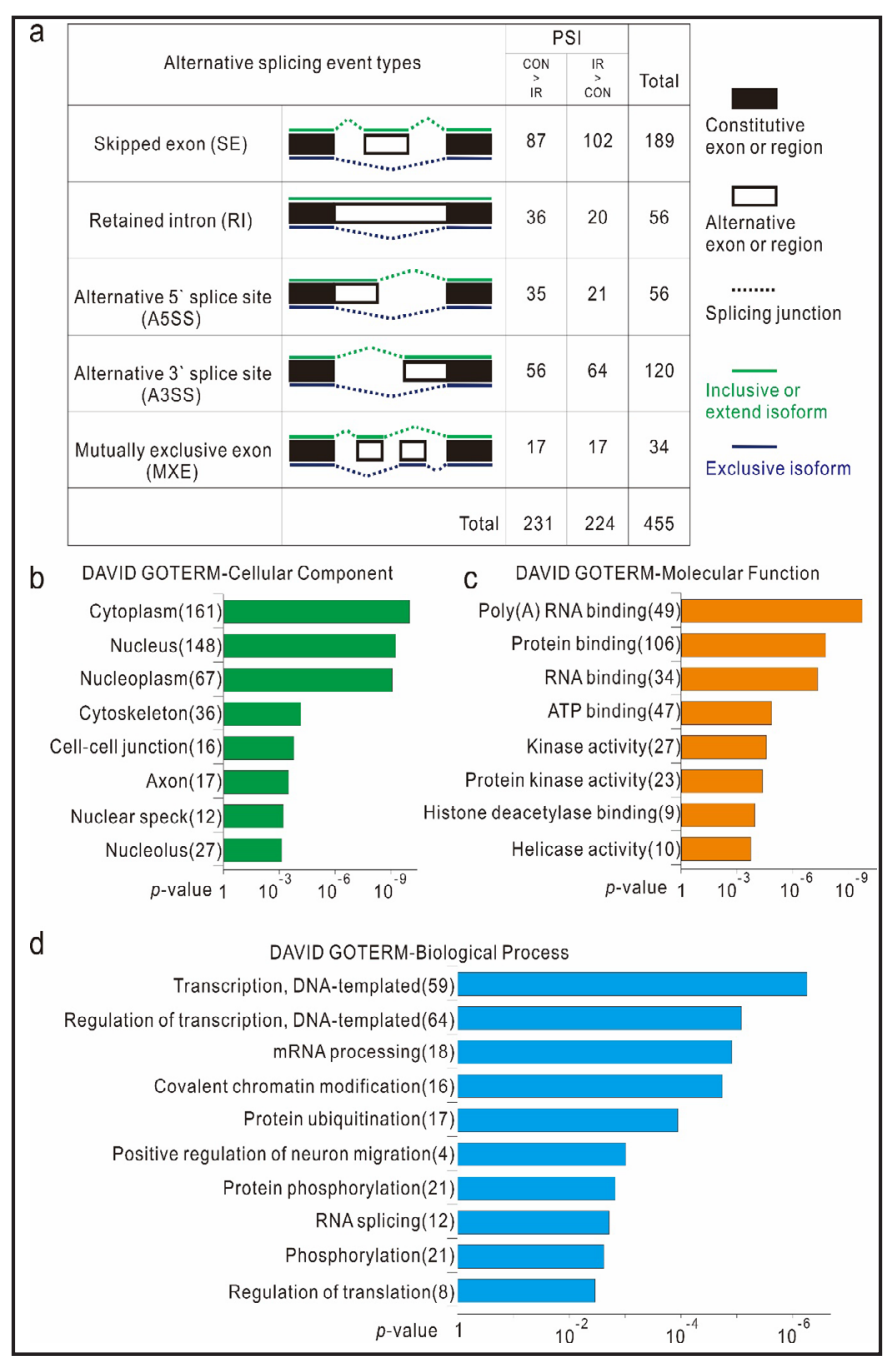

Fig. 1. AS analysis of NSCs under DNA damage conditions. (a) Number of alternatively spliced exons with higher inclusion $(|\Delta \mathrm{PSI}| \geq 10 \%$ and Bayes factor $\geq 5$, PSI of CON > IR or IR > $\mathrm{CON}$ ) for the five types of AS events between control (CON) and ionizing radiation (IR)-treated NSCs. (b-d) GO analysis of genes differentially spliced between CON and IR NSCs, showing the top ranked terms of CC (b), MF (c), and BP (d) using DAVID online tools; the numbers next to the bars represent the number of genes corresponding to that term. 


\section{Cellular Physiology Cell Physiol Biochem 2018;47:657-669 \begin{tabular}{l|l} 
DOI: 10.1159/000491893 & O 2018 The Author(s). Published by S. Karger AG, Basel \\
www.karger.com/cpb
\end{tabular} \\ Wang et al.: SIRT1 in the Alternative Splicing of Neural Stem Cells}

RNA/protein binding and the activity of enzymes such as protein kinase, histone deacetylase, and helicase (Fig. 1c). In total, 50 biological processes were affected by IR treatment with $p$ less than 0.05 , among which the most relevant were implicated in transcription, mRNA processing, and chromatin modification (Fig. 1d). These results indicated that considerable AS occurs in adult NSCs exposed to DNA damage, which influenced a large number of their biological processes.

\section{Inducible inactivation of SIRT1 in} adult NSCs

Previous work has identified SIRT1 protein expression in the nucleus of adult SVZ NSCs [32]. Using a Cre-loxP gene knockout (KO) system, we generated inducible SIRT1 KO mouse in which SIRT1 could be selectively inactivated in adult NSCs. The mice with loxP-site flanking of exon 4 of the Sirt1 gene (Fig. 2a) were crossed with NestinCreER ${ }^{T 1}$ transgenic mice, which express a tamoxifeninducible form of Cre recombinase in adult NSCs. Two-month-old NestinCreER $R^{T 1}$; Sirt $1^{\text {co/co }}$ or ${ }^{+/+}$mice were intraperitoneally injected with tamoxifen for 5 successive days (Fig. 2b); 7 days later, the mice were sacrificed for analysis. We confirmed the specific deletion of SIRT1 protein in adult SVZ NSCs and no impact on neighboring regions through SIRT1/NESTIN immunostaining (Fig. 2c). The cultured NSCs used for subsequent study were isolated from NestinCreER ${ }^{T 1}$; Sirt $1^{k o / k o}$ or ${ }^{+/+}$ mice SVZ and also validated for SIRT1 expression with immunostaining and western blotting (Fig. 2d and 2e).

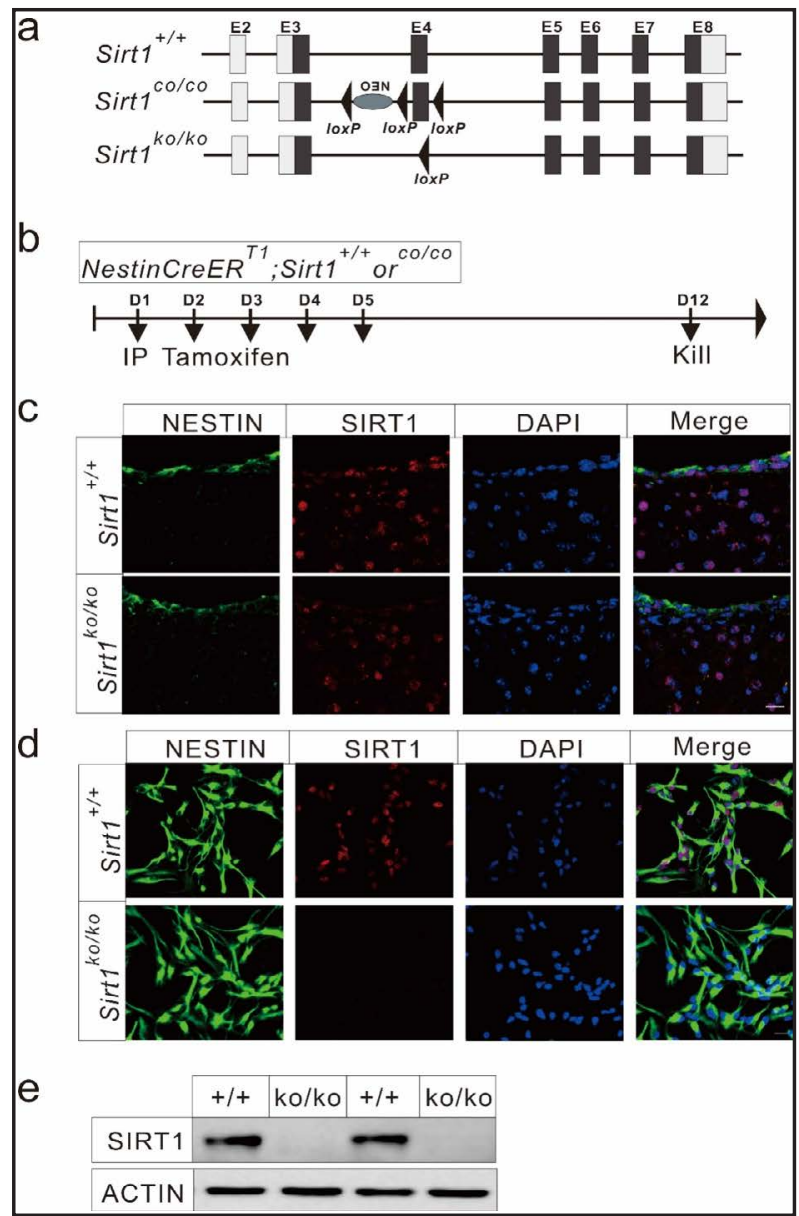

Fig. 2. Inducible inactivation of SIRT1 in adult NSCs. (a) Schematic of Sirt1 allele in wild-type (Sirt1 $1^{+/+}$), loxP floxed $\left(\operatorname{Sirt}^{\mathrm{co} / \mathrm{co}}\right)$, and Cre recombinant (Sirt1 ${ }^{\mathrm{ko} / \mathrm{ko}}$ ) mice. Exon 4 (E4) of the Sirt1 allele was floxed by loxP and spliced by Cre induction. (b) Schematic of tamoxifen-induced Sirt1 knockout in adult NSCs. Two-month-old NestinCreER ${ }^{\mathrm{T} 1}$; Sirt $1^{\text {co/co }}$ or ${ }^{+/+}$mice were intraperitoneally (IP) injected with tamoxifen for 5 successive days; 7 days later, the mice were sacrificed for analysis. (c-e) Detection of SIRT1 knockout through immunostaining of NESTIN and SIRT1 in adult Sirt1 wild-type and knockout mice NSCs in vivo (c), in vitro (d), and using western blot (e). Scale bar, $20 \mu \mathrm{m}$.

\section{SIRT1 deficiency alters the AS status of NSCs}

To investigate the impact of SIRT1 on AS in NSCs, we performed MISO analysis in wildtype (WT) and SIRT1 KO NSCs. We found that 440 exons (corresponding to 339 known genes) were differentially spliced between WT and KO NSCs, with 232 events showing higher inclusion in WT and 208 higher in KO (Fig. 3a). GO analysis revealed that the differentially spliced genes were also mainly nucleoplasm-, cytoplasm-, and nucleus-related genes, which participate in the regulation of RNA/protein binding and enzyme activity (Fig. 3b and 3c). Through GO-BP analysis, we identified 29 affected biological processes with $p$ less 
than 0.05 , among which cell cycle and cellular response to a DNA damage stimulus were known procedures influenced by SIRT1, besides the top ranked terms such as transcription regulation and chromatin modification (Fig. 3d). These results demonstrated that SIRT1 deficiency greatly altered the AS status of NSCs and thereby affected numerous cellular procedures including the cell cycle and DDR.

\section{AS analysis of SIRT1-deficient NSCs under DNA damage}

SIRT1 deficiency caused numerous AS changes in NSCs. Thus, we next compared the AS status between WT and KO NSCs under DNA damage (IR treatment). Through MISO analysis, we identified 431 exons (corresponding to 335 known genes) that were differentially spliced between WT-IR and KO-IR NSCs. KO-IR NSCs had a higher number of events with higher inclusion for all five AS types compared with WT-IR, with 186 showing higher inclusion in WT-IR and 245 higher in KO-IR (Fig. 4a). Most of the differentially spliced genes were mainly localized to the nucleus, cytoplasm, and nucleoplasm and played central roles in regulating RNA/protein binding and enzyme activity (Fig. $4 \mathrm{~b}$ and 4c). In the 45 affected biological processes with $p$ less than 0.05 , most genes were implicated in transcription, chromatin modification, and RNA processing and splicing; in particular, cell cycle regulation, the apoptotic process, and response to stress were also represented (Fig. 4d). These results also indicated the important role of SIRT1 in the DDR of NSCs at the AS level.

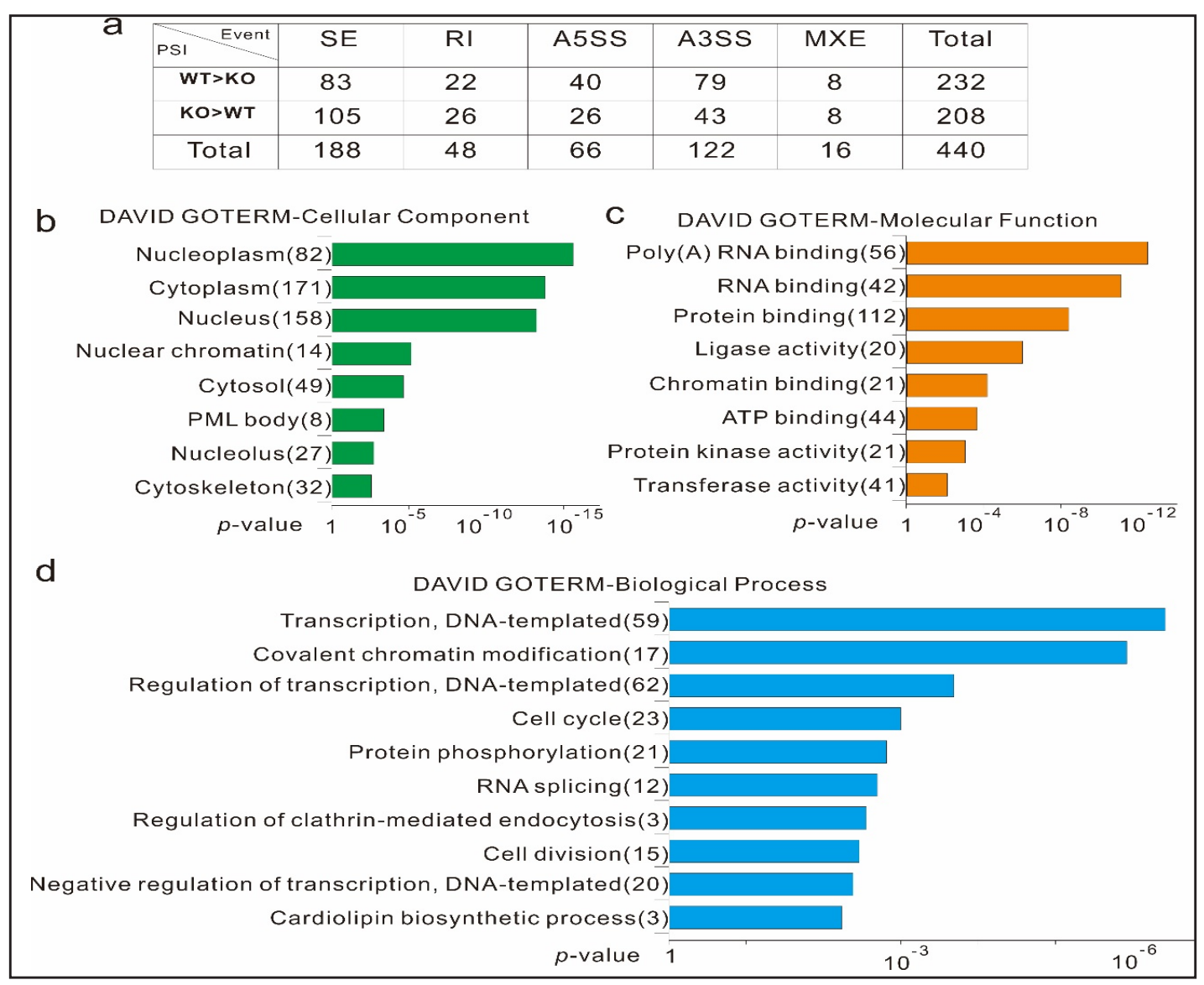

Fig. 3. AS analysis of NSCs under SIRT1 deletion conditions. (a) Number of alternatively spliced exons with higher inclusion $(|\Delta \mathrm{PSI}| \geq 10 \%$ and Bayes factor $\geq 5$, PSI of WT $>$ KO or KO $>$ WT) for the five types of AS events between SIRT1 WT and KO NSCs. (b-d) GO analysis of genes differentially spliced between WT and KO NSCs, showing the top ranked terms of CC (b), MF (c), and BP (d) using DAVID online tools; the numbers next to the bars represent the number of genes corresponding to that term. 


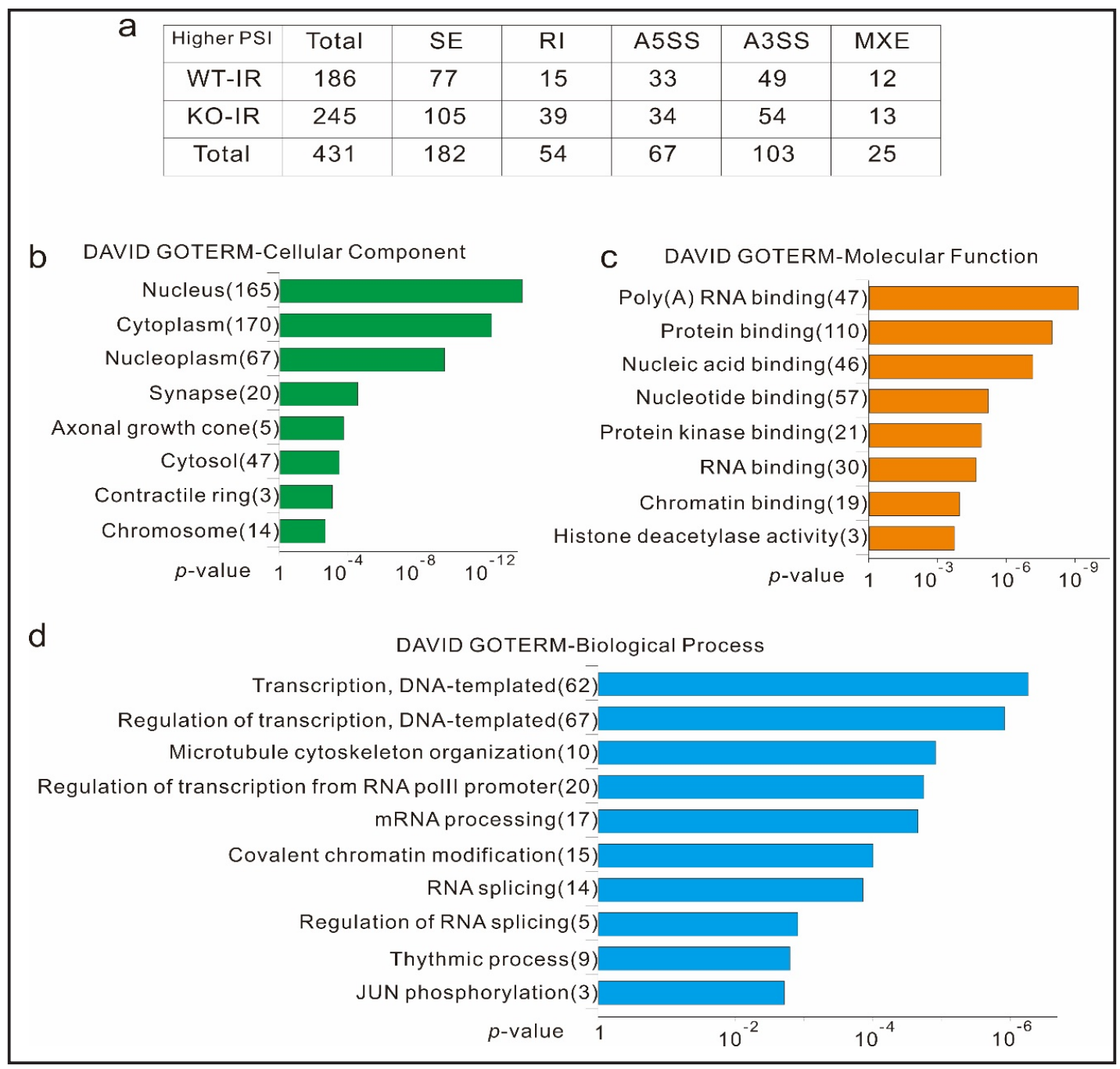

Fig. 4. AS analysis of WT and KO NSCs under IR treatment conditions. (a) Number of alternatively spliced exons with higher inclusion ( $|\Delta \mathrm{PSI}| \geq 10 \%$ and Bayes factor $\geq 5$, PSI of WT-IR $>$ KO-IR or KO-IR $>$ WT-IR) for the five types of AS events between SIRT1 WT and KO NSCs. (b-d) GO analysis of genes differentially spliced between WT-IR and KO-IR NSCs, showing the top ranked terms of CC (b), MF (c), and BP (d) using DAVID online tools; the numbers next to the bars represent the number of genes corresponding to that term.

The AS affected by SIRT1 is correlated to DNA repair and cell cycle progress in NSCS under DNA damage

To analyze the DDR-related splicing of NSCs, we compared the AS status between CON and IR (also designated as WT and WT-IR), WT and KO, and WT-IR and KO-IR. In WT and WTIR, 79 events with higher inclusion (in total, 231) and 46 with lower inclusion (in total, 224) in WT had consistent splicing status in WT vs KO; 232 had higher and 208 had lower inclusion, comprising $34 \%$ and $22 \%$, respectively ( $|\Delta \mathrm{PSI}| \geq 10 \%$ and Bayes factor $\geq 5$ ) (Fig. 5a). These results meant that SIRT1 deficiency caused numerous AS changes that correlated with the DDR. In total, there were 416 spliced exons in WT-IR and 520 in KO-IR with higher inclusion $(|\Delta \mathrm{PSI}| \geq 10 \%)$; of these, 108 and 161 events showed the same splicing status in WT vs WTIR, with successively decreased (WT $>$ WT-IR $>$ KO-IR) or increased (WT $<$ WT-IR $<$ KO-IR) PSI values (Fig. 5b). With 269 spliced exons (corresponding to 232 known genes) analyzed using GO-BP, we found that DDR-related procedures such as DNA repair, the cell cycle, and cell division were overrepresented among the 19 affected biological processes with $p$ less than 0.01 (Fig. 5c). Sashimi plots of the DNA repair-, cell cycle-, chromatin modification-, and 
RNA splicing-related events displayed successively increased or decreased expression of the spliced exons in WT vs WT-IR vs KO-IR, including all five AS types (Fig. 5d, 5e and Fig. 6).

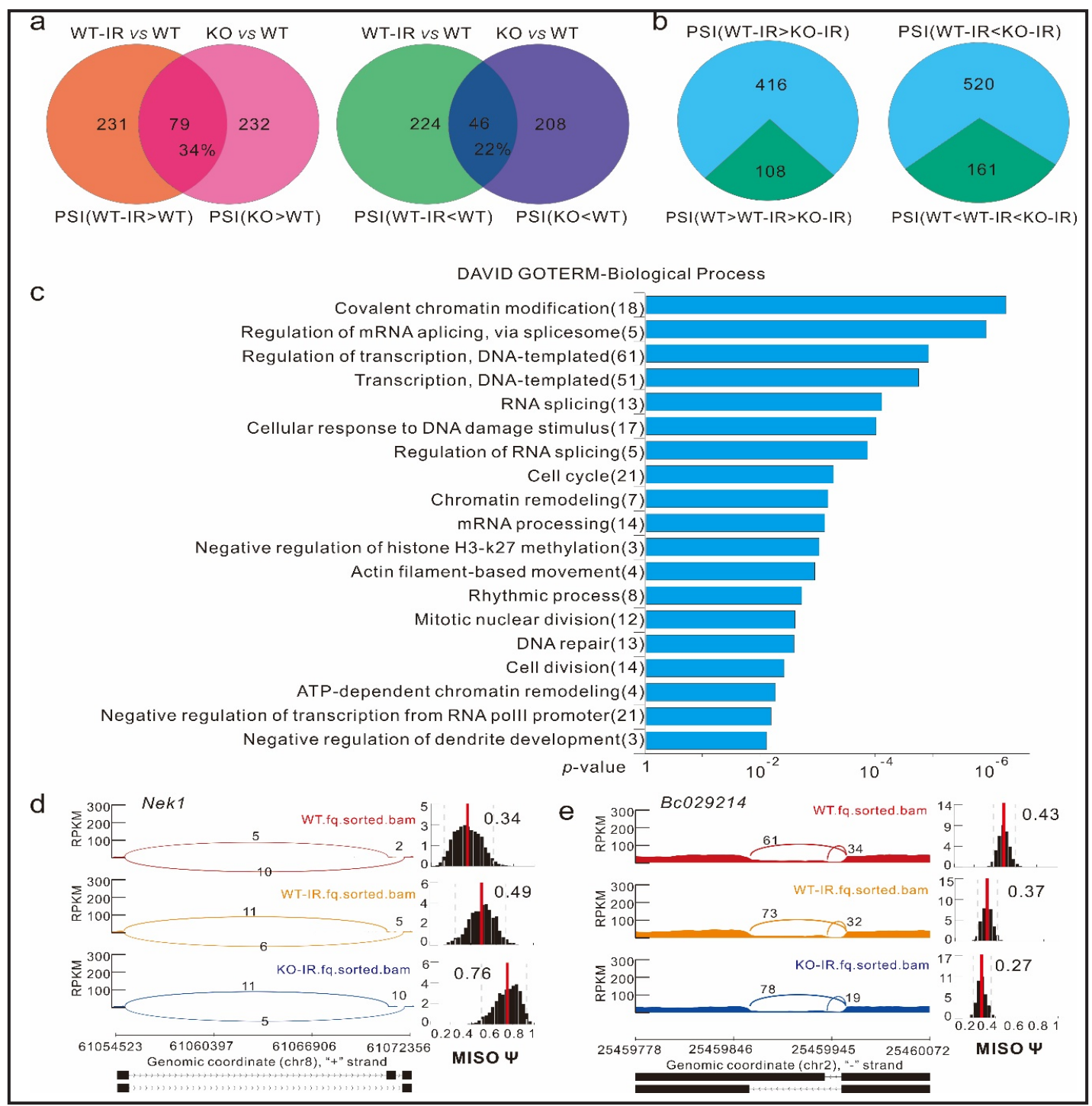

Fig. 5. Combined AS analysis in WT, KO, WT-IR, and KO-IR NSCs. (a) Number of AS events with higher ( $|\Delta \mathrm{PSI}|$ $\geq 10 \%$ and Bayes factor $\geq 5$, PSI of WT-IR $>$ WT and KO $>$ WT) or lower (PSI of WT-IR $<$ WT and KO $<$ WT) inclusion in WT NSCs displaying consistent splicing status in WT vs KO and WT vs WT-IR. (b) Number of AS events with higher or lower inclusion in WT-IR vs KO-IR $(|\Delta \mathrm{PSI}| \geq 10 \%$, PSI of WT-IR $>$ KO-IR or KO-IR $>$ WTIR), shared by WT vs KO displaying a successive PSI value increment (WT $>$ WT-IR $>$ KO-IR) or decrement (WT < WT-IR < KO-IR). (c) GO analysis of genes with a successive increment or decrement in PSI values in WT, WT-IR, and KO-IR NSCs, showing the top ranked terms of BP using DAVID online tools; the numbers next to the bars represent the number of genes corresponding to that term. (d,e) Sashimi plots of DNA repair-related genes showing the skipped exon events of Nek1 with successively increased PSI values in WT vs WT-IR vs KO-IR (d) and the alternative 5' splice site events of Bc029214 with decreased PSI values (e). 


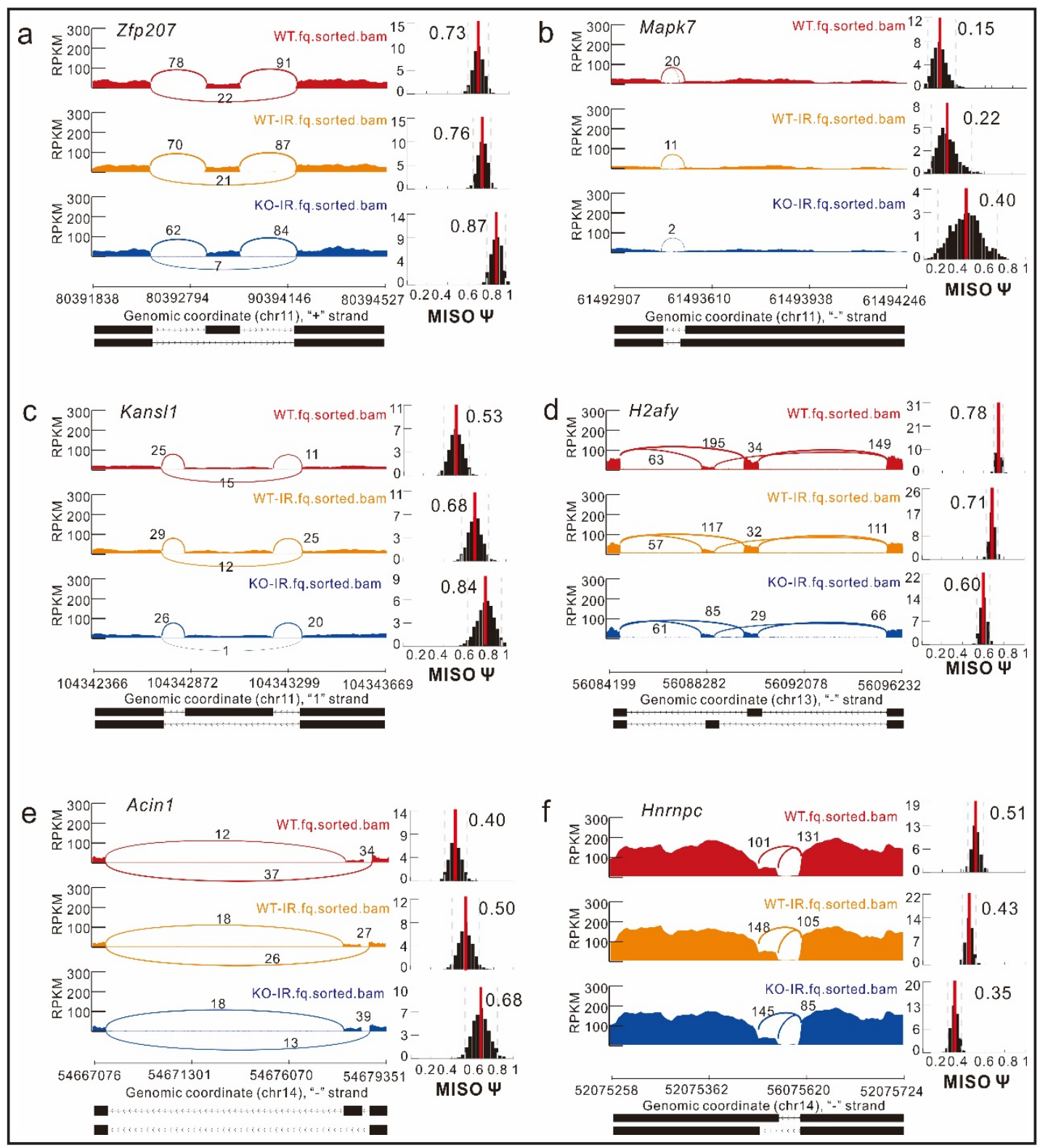

Fig. 6. Sashimi plots of genes with a successive PSI value increment or decrement in WT vs WT-IR vs KO-IR related to the cell cycle $(a, b)$, RNA splicing $(c, d)$, and chromatin modification $(e, f)$.

SIRT1 deficiency affects proliferation and DNA repair in adult NSCS

Based on the biological processes revealed by AS analysis, we examined proliferation and DNA damage repair in adult NSCs from WT and SIRT1 KO mice. Using a clone formation assay, we found that SIRT1-deficient NSCs had higher clone formation ability than WT NSCs, with neurosphere numbers of 70 in WT and 120 in KO per 1000 single cells (Fig. 7a and $7 \mathrm{~b})$. On the other hand, Ki67 immunostaining of NSCs indicated that KO NSCs had a higher proliferation rate than WT NSCs, with percentages of Ki67-positive cells in KO and WT of $72 \%$ and $62 \%$, respectively (Fig. $7 \mathrm{c}$ and $7 \mathrm{~d}$ ). All of these results demonstrated that SIRT1 deficiency plays an activating role in NSC proliferation. Immunostaining results with $\gamma \mathrm{H} 2 \mathrm{AX}$, a well-known marker of DNA damage, revealed that SIRT1 deletion increased DNA damage under normal culture conditions and decreased DNA repair under DNA damage conditions in NSCs, as determined by the $\gamma \mathrm{H} 2 \mathrm{AX}$ focal number per cell (Fig. 7e and 7f). 


\section{Cellular Physiology and Biochemistry

Fig. 7. Impact of SIRT1 knockout on proliferation and DNA repair in adult NSCs. $(a, b)$ Neurosphere formation of WT and KO NSCs (a) and the neurosphere numbers counted in both groups (b). (c,d) Ki67 immunostaining to determine the proliferation of WT and KO NSCs (c) and the quantification of Ki67 $7^{+}$cells (d). (e,f) $\gamma \mathrm{H} 2 \mathrm{AX}$ immunostaining to determine DNA damage in WT and KO NSCs under normal culture conditions (Before, 0 ) and with X-ray treatment $(1 \mathrm{~h}, 6 \mathrm{~h}, 24$ h) (e) and the quantification of $\gamma \mathrm{H}_{2} \mathrm{AX}^{+}$cells (f). Significant differences based on Student's t-test, ${ }^{*} \mathrm{p}<0.05,{ }^{* * *} \mathrm{p}<0.001$. Scale bar, $10 \mu \mathrm{m}$.
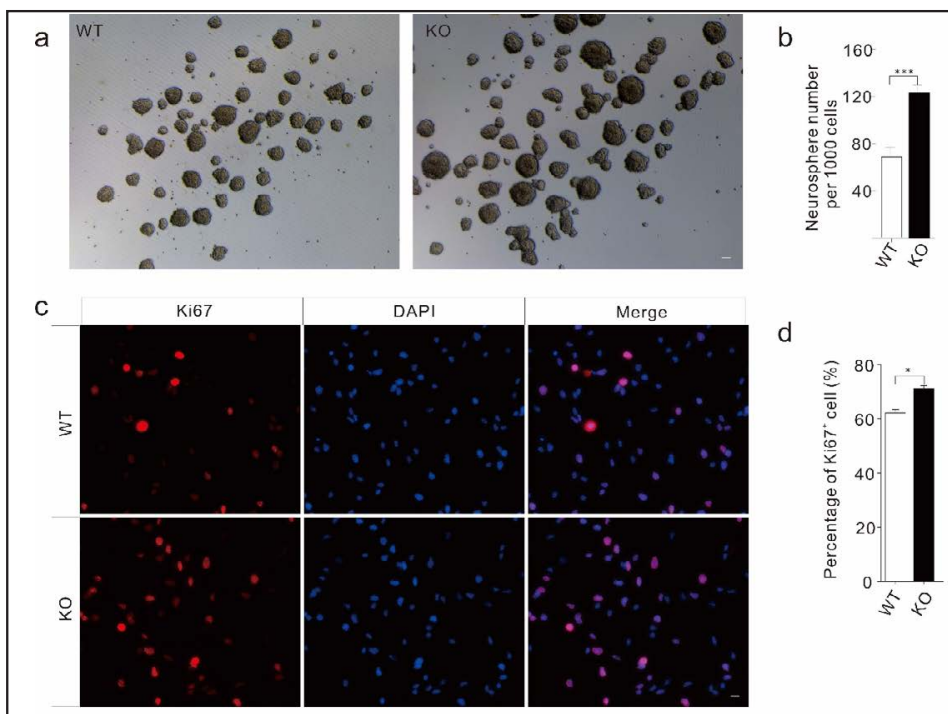

d
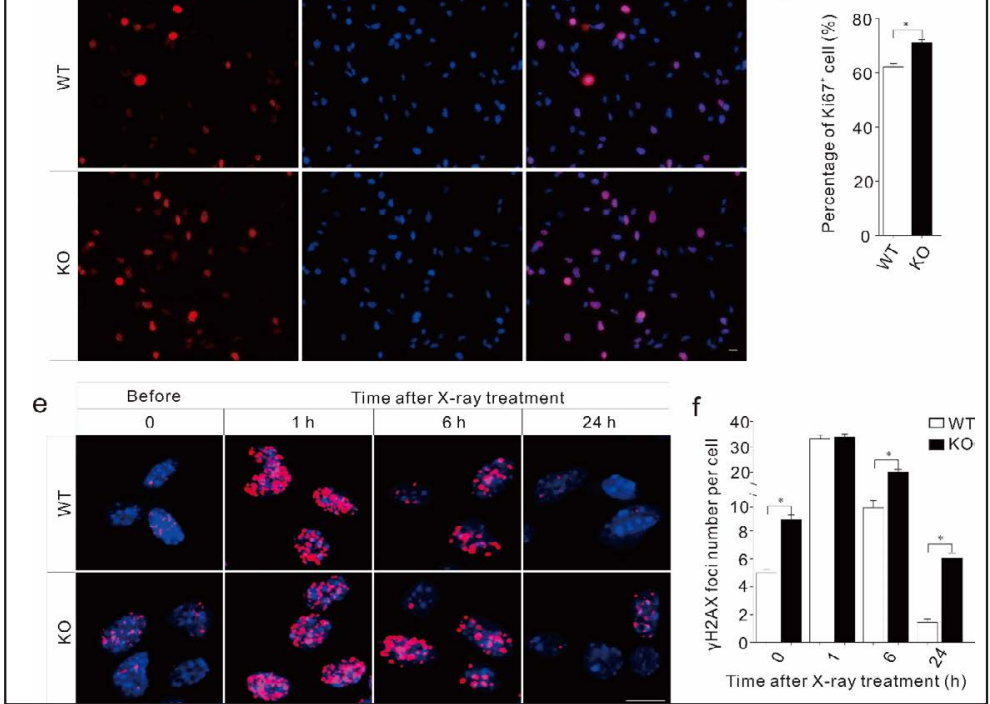

\section{Discussion}

A recent review summarizing previous studies of the RNA splicing response to DNA damage suggested that the coupling of AS to the transcription of genes involved in DDRrelated processes could be disrupted by DNA damage [15]. Specifically, studies of different cell lines treated with various DNA-damaging agents (eg, cisplatin for MCF7 cells and UV irradiation for fibroblasts) indicated extensive influence of DNA damage on AS [33]. In the present study, we performed genome-wide AS analysis of primary NSCs under IR treatment, work that has not been reported previously. Unsurprisingly, 455 differentially spliced exons and nearly 50 affected biological processes were identified between CON and IR-treated NSCs. Some were neural cell-related processes such as nervous system development, neuron projection development, dendrite morphogenesis, and negative regulation of astrocyte differentiation, which indicated that DNA damage could affect NSC development, consistent with the findings of a previous study [4]. Furthermore, the expected DDR-related procedures were also included, such as apoptotic signaling, regulation of cyclin-dependent protein kinase activity, and drug response pathways.

Given the importance of SIRT1 in cellular activities including DNA repair $[18,19]$ and the scarcity of studies concerning the impact of SIRT1 on AS, we generated SIRT1-deficient NSCs and performed AS analysis. Our work uncovered 440 differentially expressed spliced exons affected by SIRT1 deletion. These exons were enriched in 30 significant biological processes, demonstrating the prominent role of SIRT1 in AS. Previous work in NSCs revealed the involvement of SIRT1 in regulating self-renewal, differentiation, and apoptosis [20]; accordingly, our splicing analysis identified some related procedures affected by SIRT1 deletion, such as the cell cycle, cell division, and regulation of DNA-dependent DNA replication. An interesting but not completely surprising observation was that the cellular response to DNA damage was identified, suggesting that SIRT1 may influence DNA repair through the regulation of AS in NSCs. Furthermore, under DNA-damaging conditions, 


\section{Cellular Physiology Cell Physiol Biochem 2018;47:657-669 \\ \begin{tabular}{c|c} 
DOI: 10.1159/000491893 & $\begin{array}{l}\text { O 2018 The Author(s). Published by S. Karger AG, Basel } \\
\text { www.karger.com/cpb }\end{array}$
\end{tabular} \\ Wang et al.: SIRT1 in the Alternative Splicing of Neural Stem Cells}

many more processes were enriched in the DDR in WT and KO NSCs, such as responses to hydroperoxide and UV, apoptosis, cell division, and regulation of the cell cycle, revealing the considerable importance of SIRT1 in NSCs dealing with DNA damage.

In the above two comparisons (WT vs KO and WT-IR vs KO-IR), together with the analysis of CON vs IR, most of the spliced genes were overrepresented in regulation of transcription, mRNA processing, and chromatin modification, as previously noted [15, 34]. Moreover, these results are in accordance with the location of the vast majority of spliced genes in the cytoplasm, nucleus, and nucleoplasm, reflecting their RNA/protein binding and enzyme activity functions, because chromatin is intimately associated with the control of splice site selection and coordinates DNA repair activities.

To further explore the DDR affected by SIRT1 deletion at the AS level, we performed combined analyses of AS events occurring in adult NSCs. Within the WT vs KO comparison, $34 \%$ of spliced exons with higher inclusion in WT and 22\% with higher inclusion in KO displayed a consistent splicing status in CON vs IR, which confirmed the ability of SIRT1 deficiency alone to cause changes in AS involved in the DDR in NSCs responding to endogenous stimuli. In WT-IR vs KO-IR, 26\% and 31\%, respectively, of spliced events showing higher inclusion shared a similar splicing status with CON vs IR; DDR-related processes such as the cellular response to DNA damage stimuli, cell cycle, cell division, and DNA repair were identified among the shared spliced exons. These findings further indicated the important role of SIRT1 in modulating the DDR of NSCs under exogenous stress.

Our analyses identified genes involved in DNA damage repair (eg, Rad51ap1, Nek1, Lig3) and correlated with cell cycle control (eg, Zfp207, Mapk7) in WT vs WT-IR vs K0-IR as determined by the PSI value and expressed with sashimi plots. As verified in recent work, the RAD51AP1-UAF1 complex promotes RAD51-mediated homologous DNA pairing [35]; NEK1 can orchestrate homologous recombination and replication fork stability by regulating RAD54 [36]; and MAPK7 plays a key role in controlling cell proliferation, migration, and invasion in osteosarcoma [37]. We also performed further experiments to validate the phenotypic changes in NSCs caused by SIRT1 deletion, with neurosphere formation and Ki67 immunostaining results demonstrating the inactivating role of SIRT1 in proliferation and $\gamma \mathrm{H} 2 \mathrm{AX}$ staining data revealing the need for SIRT1 in DNA damage repair. All of these results provide strong evidence to support our belief that SIRT1 may regulate the DDR in NSCs by controlling AS.

In conclusion, in the present study, we profiled the AS of adult NSCs under DNA damage conditions and with SIRT1 deficiency. Our findings revealed widespread AS in adult NSCs responding to DNA damage and massive biological processes affected by SIRT1 at the AS level. In NSCs, an overrepresentation of splicing changes occurred in genes involved in regulation of transcription, chromatin modification, RNA processing, and splicing. In particular, we uncovered the DDR-related processes such as cell cycle control and DNA repair affected by SIRT1 deficiency. Primarily, we showed that the significant role of SIRT1 in the DDR of NSCs could involve the regulation of AS. Our work provides a novel insight into the mechanisms underlying DDR in stem cells. However, given the preliminary findings, further detailed studies need to be performed.

\section{Acknowledgements}

This work was supported by The National Major Scientific and Technological Special Project for "Significant New Drugs Development" (2018ZX09201002-005); the Ministry of Science and Technology of China (2016YFE0107200); the National Natural Science Foundation of China $(31471029,31671055,81461138037,31571058,21671150$, 31371497, 81400111, 31601102, 81401053); Xu Jun's expert work station (2017IC025); Shanghai Easter Scholar (8101219003, Z.G.); and the Fundamental Research Fund for the Central Universities (1500219107, J.X.). 


\section{Cellular Physiology Cell Physiol Biochem 2018;47:657-669 \begin{tabular}{l|l} 
DOI: 10.1159/000491893 & O 2018 The Author(s). Published by S. Karger AG, Basel \\
www.karger.com/cpb
\end{tabular}}

Wang et al.: SIRT1 in the Alternative Splicing of Neural Stem Cells

\section{Disclosure Statement}

The author(s) declare no competing financial interests.

\section{References}

Dong CM, Wang XL, Wang GM, Zhang WJ, Zhu L, Gao S, Yang DJ, Qin Y, Liang QJ, Chen YL, Deng HT, Ning $\mathrm{K}$, Liang AB, Gao ZL, Xu J: A stress-induced cellular aging model with postnatal neural stem cells. Cell Death Dis 2014;5:e1116.

Etienne 0, Bery A, Roque T, Desmaze C, Boussin FD: Assessing cell cycle progression of neural stem and progenitor cells in the mouse developing brain after genotoxic stress. J Vis Exp 2014. Doi: $10.3791 / 51209$ Acharya MM, Lan ML, Kan VH, Patel NH, Giedzinski E, Tseng BP, Limoli CL: Consequences of ionizing radiation-induced damage in human neural stem cells. Free Radic Biol Med 2010;49:1846-1855. Tanori M, Pasquali E, Leonardi S, Casciati A, Giardullo P, De Stefano I, Mancuso M, Saran A, Pazzaglia S: Developmental and oncogenic radiation effects on neural stem cells and their differentiating progeny in mouse cerebellum. Stem Cells 2013;31:2506-2516. Schneider L, Pellegatta S, Favaro R, Pisati F, Roncaglia P, Testa G, Nicolis SK, Finocchiaro G, d'Adda di Fagagna F: DNA damage in mammalian neural stem cells leads to astrocytic differentiation mediated by BMP2 signaling through JAK-STAT. Stem Cell Reports 2013;1:123-138. Barazzuol L, Jeggo PA: In vivo sensitivity of the embryonic and adult neural stem cell compartments to low-dose radiation. J Radiat Res 2016;57:i2-i10. Barazzuol L, Rickett N, Ju L, Jeggo PA: Low levels of endogenous or X-ray-induced DNA double-strand breaks activate apoptosis in adult neural stem cells. J Cell Sci 2015;128:3597-3606.

Zou Y, Zhang N, Ellerby LM, Davalos AR, Zeng X, Campisi J, Desprez PY: Responses of human embryonic stem cells and their differentiated progeny to ionizing radiation. Biochem Biophys Res Commun 2012;426:100-105.

Beli P, Lukashchuk N, Wagner SA, Weinert BT, Olsen JV, Baskcomb L, Mann M, Jackson SP, Choudhary C: Proteomic investigations reveal a role for RNA processing factor THRAP3 in the DNA damage response. Mol Cell 2012;46:212-225.

10 Colla S, Ong DS, Ogoti Y, Marchesini M, Mistry NA, Clise-Dwyer K, Ang SA, Storti P, Viale A, Giuliani N, Ruisaard K, Ganan Gomez I, Bristow CA, Estecio M, Weksberg DC, Ho YW, Hu B, Genovese G, Pettazzoni P, Multani AS, Jiang S, Hua S, Ryan MC, Carugo A, Nezi L, Wei Y, Yang H, D’Anca M, Zhang L, Gaddis S, Gong T, Horner JW, Heffernan TP, Jones P, Cooper LJ, Liang H, Kantarjian H, Wang YA, Chin L, Bueso-Ramos C, Garcia-Manero G, DePinho RA: Telomere dysfunction drives aberrant hematopoietic differentiation and myelodysplastic syndrome. Cancer Cell 2015;27:644-657.

11 Busa R, Geremia R, Sette C: Genotoxic stress causes the accumulation of the splicing regulator Sam68 in nuclear foci of transcriptionally active chromatin. Nucleic Acids Res 2010;38:3005-3018.

12 Nakka KK, Chaudhary N, Joshi S, Bhat J, Singh K, Chatterjee S, Malhotra R, De A, Santra MK, Dilworth FJ, Chattopadhyay S: Nuclear matrix-associated protein SMAR1 regulates alternative splicing via HDAC6mediated deacetylation of Sam68. Proc Natl Acad Sci U S A 2015;112:E3374-3383. Pelisch F, Pozzi B, Risso G, Munoz MJ, Srebrow A: DNA damage-induced heterogeneous nuclear ribonucleoprotein $\mathrm{K}$ sumoylation regulates p53 transcriptional activation. J Biol Chem 2012;287:30789-30799.

14 Wong A, Zhang S, Mordue D, Wu JM, Zhang Z, Darzynkiewicz Z, Lee EY, Lee MY: PDIP38 is translocated to the spliceosomes/nuclear speckles in response to UV-induced DNA damage and is required for UVinduced alternative splicing of MDM2. Cell Cycle 2013;12:3184-3193.

15 Shkreta L, Chabot B: The RNA Splicing Response to DNA Damage. Biomolecules 2015;5:2935-2977.

16 Paulsen RD, Soni DV, Wollman R, Hahn AT, Yee MC, Guan A, Hesley JA, Miller SC, Cromwell EF, SolowCordero DE, Meyer T, Cimprich KA: A genome-wide siRNA screen reveals diverse cellular processes and pathways that mediate genome stability. Mol Cell 2009;35:228-239.

17 Wan Y, Zheng X, Chen H, Guo Y, Jiang H, He X, Zhu X, Zheng Y: Splicing function of mitotic regulators links R-loop-mediated DNA damage to tumor cell killing. J Cell Biol 2015;209:235-246. 


\section{Cellular Physiology Cell Physiol Biochem 2018;47:657-669 \begin{tabular}{l|l} 
DOI: 10.1159/000491893 & $\begin{array}{l}\text { O } 2018 \text { The Author(s). Published by S. Karger AG, Basel } \\
\text { www.karger.com/cpb }\end{array}$
\end{tabular} \\ Wang et al.: SIRT1 in the Alternative Splicing of Neural Stem Cells}

18

19

$-20$

$-21$

$-22$

Libert S, Guarente L: Metabolic and neuropsychiatric effects of calorie restriction and sirtuins. Annu Rev Physiol 2013;75:669-684.

Haigis MC, Sinclair DA: Mammalian sirtuins: biological insights and disease relevance. Annu Rev Pathol 2010;5:253-295.

Cai Y, Xu L, Xu H, Fan X: SIRT1 and Neural Cell Fate Determination. Mol Neurobiol 2016;53:2815-2825.

Li K, Casta A, Wang R, Lozada E, Fan W, Kane S, Ge Q Gu W, Orren D, Luo J: Regulation of WRN protein cellular localization and enzymatic activities by SIRT1-mediated deacetylation. J Biol Chem 2008;283:7590-7598.

Jeong J, Juhn K, Lee H, Kim SH, Min BH, Lee KM, Cho MH, Park GH, Lee KH: SIRT1 promotes DNA repair activity and deacetylation of Ku70. Exp Mol Med 2007;39:8-13.

Yuan Z, Zhang X, Sengupta N, Lane WS, Seto E: SIRT1 regulates the function of the Nijmegen breakage syndrome protein. Mol Cell 2007;27:149-162.

Oberdoerffer P, Michan S, McVay M, Mostoslavsky R, Vann J, Park SK, Hartlerode A, Stegmuller J, Hafner A, Loerch P, Wright SM, Mills KD, Bonni A, Yankner BA, Scully R, Prolla TA, Alt FW, Sinclair DA: SIRT1 redistribution on chromatin promotes genomic stability but alters gene expression during aging. Cell 2008;135:907-918.

Zannini L, Buscemi G, Kim JE, Fontanella E, Delia D: DBC1 phosphorylation by ATM/ATR inhibits SIRT1 deacetylase in response to DNA damage. J Mol Cell Biol 2012;4:294-303.

Conrad E, Polonio-Vallon T, Meister M, Matt S, Bitomsky N, Herbel C, Liebl M, Greiner V, Kriznik B, Schumacher S, Krieghoff-Henning E, Hofmann TG: HIPK2 restricts SIRT1 activity upon severe DNA damage by a phosphorylation-controlled mechanism. Cell Death Differ 2016;23:110-122.

Liu T, Lin YH, Leng W, Jung SY, Zhang H, Deng M, Evans D, Li Y, Luo K, Qin B, Qin J, Yuan J, Lou Z: A divergent role of the SIRT1-TopBP1 axis in regulating metabolic checkpoint and DNA damage checkpoint. Mol Cell 2014;56:681-695.

Guo W, Patzlaff NE, Jobe EM, Zhao X: Isolation of multipotent neural stem or progenitor cells from both the dentate gyrus and subventricular zone of a single adult mouse. Nat Protoc 2012;7:2005-2012. Kim D, Langmead B, Salzberg SL: HISAT: a fast spliced aligner with low memory requirements. Nat Methods 2015;12:357-360.

Katz Y, Wang ET, Airoldi EM, Burge CB: Analysis and design of RNA sequencing experiments for identifying isoform regulation. Nat Methods 2010;7:1009-1015.

Huang da W, Sherman BT, Lempicki RA: Systematic and integrative analysis of large gene lists using DAVID bioinformatics resources. Nat Protoc 2009;4:44-57.

Rafalski VA, Ho PP, Brett JO, Ucar D, Dugas JC, Pollina EA, Chow LM, Ibrahim A, Baker SJ, Barres BA, Steinman L, Brunet A: Expansion of oligodendrocyte progenitor cells following SIRT1 inactivation in the adult brain. Nat Cell Biol 2013;15:614-624.

Gabriel M, Delforge Y, Deward A, Habraken Y, Hennuy B, Piette J, Klinck R, Chabot B, Colige A, Lambert C: Role of the splicing factor SRSF4 in cisplatin-induced modifications of pre-mRNA splicing and apoptosis. BMC Cancer 2015;15:227.

Ip JY, Schmidt D, Pan Q Ramani AK, Fraser AG, Odom DT, Blencowe BJ: Global impact of RNA polymerase II elongation inhibition on alternative splicing regulation. Genome Res 2011;21:390-401. Liang F, Longerich S, Miller AS, Tang C, Buzovetsky O, Xiong Y, Maranon DG, Wiese C, Kupfer GM, Sung P: Promotion of RAD51-Mediated Homologous DNA Pairing by the RAD51AP1-UAF1 Complex. Cell Rep 2016;15:2118-2126.

Spies J, Waizenegger A, Barton O, Surder M, Wright WD, Heyer WD, Lobrich M: Nek1 Regulates Rad54 to Orchestrate Homologous Recombination and Replication Fork Stability. Mol Cell 2016;62:903-917. Tesser-Gamba F, Lopes LJ, Petrilli AS, Toledo SR: MAPK7 gene controls proliferation, migration and cell invasion in osteosarcoma. Mol Carcinog 2016;55:1700-1713. 